\title{
Introducing the Lettera Matematica International Edition
}

\author{
Renato Betti · Angelo Guerraggio $\cdot$ Settimo Termini
}

Published online: 22 May 2013

(c) Centro P.RI.ST.EM, Università Commerciale Luigi Bocconi 2013

\begin{abstract}
The editors of the Lettera Matematica International Edition introduce the new journal and explain its scope and aims.
\end{abstract}

Keywords Lettera Matematica $\cdot$ Pristem $\cdot$ Mathematical culture

The Lettera Matematica PRISTEM is a quarterly journal for mathematical culture which began publication in Italy in 1991 and has been published by Springer since 1997. PRISTEM is the acronym for a historical-mathematical research group based at Milan's Università Bocconi. The group founded the journal and still directs it. Its editorial board has grown over the years to include mathematicians, historians of mathematics, and scholars from various Italian universities, and recently from some French universities as well.

\footnotetext{
R. Betti

Dipartimento di Matematica, Politecnico di Milano, Piazza Leonardo da Vinci 32, 20133 Milan, Italy

e-mail: renato.betti@polimi.it

A. Guerraggio $(\square)$

Università Bocconi di Milano, Via Sarfatti 25,

20136 Milan, Italy

e-mail: angelo.guerraggio@unibocconi.it

S. Termini

Dipartimento di Matematica e Informatica, Università degli studi

di Palermo, Via Archirafi, 34, 90123 Palermo, Italy

e-mail: settimo.termini@gmail.com
}

Each issue of the journal is traditionally divided into four sections: one related to current events and news items from the world of mathematics; one which is largely devoted to increasing awareness of mathematics (divulgation); a third of a historic nature; and a fourth related to problems of teaching and learning mathematics. For some time now, these 'normal' issues of the Lettera Matematica have been flanked each year by a special, monographic issue devoted to a special figure or theme in mathematics.

It is precisely here that the journey of the newly founded Lettera Matematica International Edition begins, with a double issue dedicated to Henri Poincaré. This first issue corresponds to issue $84-85$ of the Italian edition, which will continue its publication in tamdem with the international edition. The new LMIE is of the same nature as its Italian forerunner: a journal that is sufficiently 'curious' about other worlds that come into contact more or less directly with that of mathematics, without being hindered by the disciplinary boundaries that often turn out to be artificial or groundless. Here you will find articles that refer explicitly to mathematical research and its technological applications, as well as others that regard physics and its history, society, educational questions, and scientific policies. These are aimed not only at professional mathematicians, but at teachers, students and those who are not mathematicians but who are, like us, sufficiently curious about the beauty and usefulness of the scientific world. The aim is to arrive at concentric circles that are increasingly large, using simple language - that is, as simple as the complexity of the arguments themselves allows-with the assistance of images where necessary.

These are the hopes of the editors as they inaugurate the Lettera Matematica International Edition. It will then be 
up to the readers to tell us how close we have come to achieving our aims. We look forward to hearing your suggestions and ideas. Thank you.

\section{Author Biographies}

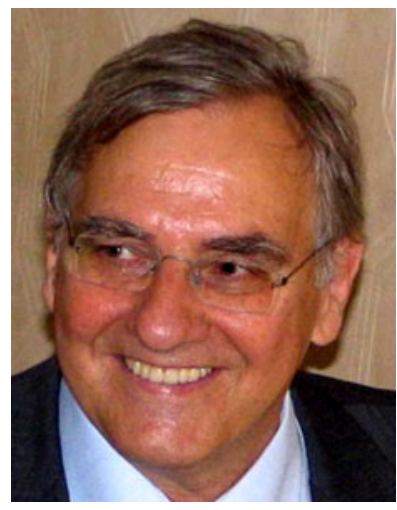

Renato Betti is a former professor of geometry at the Politecnico di Milano, where he is currently Adjunct Professor. His research concerns category theory and its applications to algebra and geometry. His recent publications include $L a$ matematica come abitudine del pensiero. Le idee scientifiche di Pavel Florenskij (Libri del Pristem, 2009). He is a corresponding member of the Accademia Nazionale Virgiliana in Mantua.

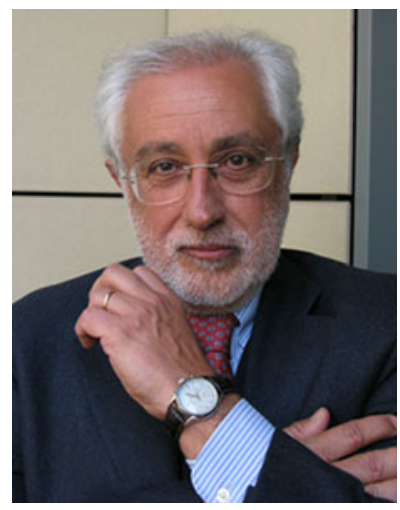

Angelo Guerraggio is a Full Professor of Mathematics for the Economy at the Università Bocconi in Milan and the Università dell'Insubria in Varese. His research concerns with non-linear programming and history of mathematics. He is the Coordinator of the Centro PRISTEM at the Università Bocconi, and Editor-inChief of the Lettera matematica PRISTEM. He has written numerous books, including the most recent 15 grandi idee matematiche (Bruno Mondadori, 2013).

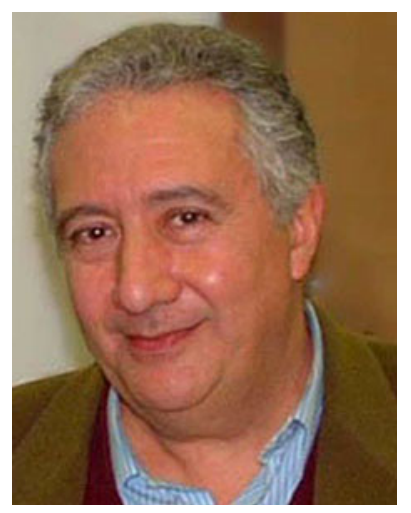

Settimo Termini is Full Professor of Informatica teorica at the University of Palermo and an Affiliated Researcher of the European Center for Soft Computing (ECSC) in Mieres (Asturie), Spain. From 2002 to 2009 he was director of the Istituto di Cibernetica 'Eduardo Caianiello' of the Italian National Research Council (CNR) in Naples. A physicist by training, he is primarily concerned with problems raised by the treatment of incomplete and revisable information in complex systems. He was one of the first in Italy to introduce topics related to fuzzy systems, giving the start, together with Aldo de Luca, to the theory of fuzziness measures. He has analysed several of the epistemological problems that arise in this context and, more recently, the relationship between scientific research and economic development. His publications include Imagination and Rigor (Springer, 2006), Contro il declino (co-authored with Pietro Greco, Codice edizioni, 2007), Memoria e Progetto (coedited with Pietro Greco, Edizioni GEM, 2010). 